\title{
Şanlıurfa Popülasyonunda Maksiller Sinüs Septa Morfoloji ve İnsidansının Retrospektif Olarak Değerlendirilmesi
}

\section{Retrospective Evaluation of The Maxillary Sinus Septa Morphology and It's Incidence in the Şanliurfa Population}

\author{
$\underline{\text { Halil İbrahim DURMUS }^{1}}$
}

1 Harran Üniversitesi Diş Hekimliği Fakültesi Ağız, Diş ve Çene Cerrahisi A.B.D.

Öz.

Amac: Bu çalısmanın amacı; maksiller sinüste septa görülme sıklığını, septa sayııını, konumunu ve septanın maksiller sinüste yönelimini bilgisayarlı tomografi ile tespit etmektir. Ayrıca, sinus septasının hasta ile ilgili faktörleri olan yaş, cinsiyet ve dentisyon tipi ile ilişkisini değerlendirmektir.

Materyal ve metod: Bu retrospektif çalışma Harran Üniversitesi Diş Hekimliği Fakültesi hastanesine Ocak 2017-Ocak 2020 tarihleri arasında başvuran hastalardan alınmış görüntüler incelenerek yapıldı. Çalışmada etik kurul onayı Harran Üniversitesi Klinik Araştırmalar Etik Kurulu'dan HRU/20.09.08 sayııı kararı ile alınmıştır. Çalışma 500 adet baş boyun bölgesi bilgisayarlı tomografi görüntüsü değerlendirilerek gerçekleştirildi. Görüntüler incelenirken her hasta için, hastanın yaşı, cinsiyeti, hastanın dişli-kısmi dişli-tam dişsiz oluşu parametreleri kaydedildi. Septalar koronal, sagital ve transversal olmak üzere sınıflandırıldı. Bulgular: Toplam 500 adet bilgisayarlı tomografi görüntüsü incelendi. Bunların 446 tanesi dahil edilme kriterlerine uyduğundan çalışmaya dahil edildi. Böylece toplam 892 tane maksiller sinüs incelendi. Incelenen hastaların 198'i $(\% 44,4)$ erkek ve 248 'i $(\% 55,6)$ kadındı. Hastaların ortalama yaşı 33.4, en küçüğü 18, en büyüğü 79 yaşındaydı. Hastaların 248'i ( $\% 55,6)$ tam dișli, 168 'i $(\% 37,7)$ kısmi dişli ve 30'u (\%6.7) tam dişsizdi. 135 $(\% 30,2)$ hastada en az bir septum tespit edildi. Bu hastaların 126 'sında $(\% 93,3)$ sadece bir septum görülürken, 9'unda (\% 6.7) birden fazla septum görüldü. Hastalarda toplam 146 adet septum tespit edildi. Maksiller sinüs septası prevelansı ile hastaların yaşı $(p=0.415)$, cinsiyeti $(p=0.443)$ ve dentisyon tipi $(p=0.078)$ arasında anlamlı bir ilişki bulunamamıştır. Maksiller sinüs septasının yönelim ve konumları arasında anlamlı bir ilişki bulunmuştur ( $p=$ 0.017).

Sonuç: Bu çalışma sonucunda popülasyonun yaklaşık üçte birinde maksiler sinüs septası olduğu görüldü. Yapılacak implant cerrahilerinin üç boyutlu görüntülerle planlanması yaşanabilecek komplikasyonların azalmasını sağlamaktadır. Ayrıca üç boyutlu görüntülerin iki boyutlu görüntülere dayalı planlamaları değiştirebileceği görüldü. Ancak bu çalışma sonuçlarının yapılacak daha kapsamlı çalışmalarla doğrulanmasına ihtiyaç olduğu değerlendirildi.

Anahtar Kelimeler: Maksiler sinüs, Dental implant, Maksiler sinüs septası

Abstract

Background: The purpose of this study is to determine the frequency, number, location and the orientation of the septa in the maxillary sinus by computed tomography. In addition, to evaluate the relationship of sinus septa with age, gender and type of dentition, which are patient related factors.

Materials and Methods: This retrospective study was done by examining the images taken from patients who applied to Harran University Faculty of Dentistry between January 2017 and January 2020. Ethics committee approval was obtained from Harran University Clinical Research Ethics Committee with the decision numbered HRU / 20.09.08. The study was carried out by evaluating the computed tomography images of 500 head and neck regions. While examining the images, the parameters of the patient's age, gender and status of the dentition (full dentition, partial dentition, or edentulous) were recorded. Septas were classified as coronal, sagittal and transversal.

Results: A total of 500 computed tomography images were examined. 446 of them were included in the study because they met the inclusion criteria. Thus, a total of 892 maxillary sinuses were examined. 198 (44.4\%) of the patients examined were male and $248(55.6 \%)$ were female. The average age of the patients was 33.4 years old, the youngest was 18 and the oldest was 79 years old. 248 patients (55.6\%) were full dentition, $168(37.7 \%)$ were partial dentition and $30(6.7 \%)$ were edentulous. At least one septum was detected in $135(30.2 \%)$ patients. $126(93.3 \%)$ of these patients had only one septum, while $9(6.7 \%)$ had more than one septum. A total of 146 septums were detected in the patients. No significant relation was found between the prevalence of maxillary sinus septa and the age $(p=0.415)$, gender $(p=0.443)$ and dentition type $(p=0.078)$ of the patients. A significant relation was found between the orientation and positions of the maxillary sinus septa $(p=0.017)$.

Conclusion: As a result of this study, it was observed that approximately one third of the population had a maxillary sinus septa. Planning implant surgeries with three-dimensional images reduces the possible complications. It was also seen that threedimensional images could change the planning based on two-dimensional images. However, it was evaluated that the results of these study need to be confirmed by more comprehensive studies.

Key words: Maxillary sinus, Dental implant, Maxillary sinus septa

\section{Sorumlu Yazar I \\ Corresponding Author}

Dr.Öğr. Üyesi Halil librahim DURMUŞ

Harran Üniversitesi Diş Hekimliği Fakültesi Hamidiye Mah. Yenişehir Kampüsü

Haliliye / Şanlıurfa / Türkiye

Tel: 05426692702

Fax: 0 (274) 2652285

Email: durmushibrahim@gmail.com

Geliş tarihi / Received:

08.06.2020

Kabul tarihi / Accepted:

08.07.2020

DOI: $10.35440 /$ hutfd. 716450 


\section{Giriş}

İmplantlar, son yıllarda kısmi ve total diş eksikliklerinin rehabilitasyonunda oldukça yaygın kullanılmaya başlanmıştır(1).Maksilla posterior bölgedeki dişlerin kaybedilmesi ile osteoklastik aktivite artar ve alveolar sırt atrofiye olur. Vertikal yöndeki bu kemik kaybı maksillada implant planlamasını komplike hale getirir(2). Bu alanda kemik kalınlığını artırmak için maxiller sinüs tabanının yükseltilmesine intiyaç duyulur $(3,4)$. Maksiller sinüs anatomisi, özelikle de maxiller sinus septasının oluşu,yapılan sinüs cerrahilerini etkilemektedir (5-7). Maksiller sinüs septası; kortikal kemiğin ince yapılarıdır. Arthur S. Underwood septayı şöyle tanımlamıştır: maxiller sinüs septası ince, kırılgan ve orak şekilli kemik duvarlarıdır(8). Septa, sinüsü ikiye veya daha fazla bölmeye bölebilir $(9,10)$. Maksiller sinüsün morfolojisi kişinin yaşından, dişilerinin anatomisinden ve diş eksikliklerinden etkilenebilir (11). Gelişimsel olarak septa, ikiye ayrilır: primer septa konjenital çeşitlilikten kaynaklanır ve yüzün gelişimi sırasında oluşur. Sekonder septa ise diş kaybı veya maksiller sinüs duvarlarının düzensiz havalanmasından kaynaklı daha sonra gelişebilir $(9,10,12)$.

Yapılan çalışmalar; maksiller sinüs lift cerrahisinde maksiler septa varlığı ve Schneiderian membranı perforasyonu arasında bir ilişki olduğunu göstermiştir. Çünkü Schneiderian membranı kemiğe sinüs septası boyunca çok daha sıkı bağlanmıştı $(5,6)$. Bu bölgelere yapılacak Implant uygulamalarının bilgisayarlı tomografi ile planlanması, septa varlığı durumunda olası komplikasyonlar tespit edilebilir ve bu riskler cerrahi sırasında ve sonrasında gözönüne alınabilir $(7,13)$.

Bu çalışmanın amacı; maksiller sinüste septa görülme sıkIığını, septa sayısını, konumunu ve septanın maksiller sinüste yönelimini bilgisayarlı tomografi ile tespit etmektir. Ayrıca, sinus septasının hasta ile ilgili faktörleri olan yaş, cinsiyet ve dentisyon tipi ile ilişkisini değerlendirmektir.

\section{Materyal ve Metod}

Bu retrospektif çalışma; Harran Üniversitesi Diş Hekimliği Fakültesi hastanesine ocak 2017- ocak 2020 tarihleri arasında başvuran hastalardan alınmış görüntüler incelenerek yapılmıştır. Çalışmada etik kurul onayı Harran Üniversitesi Klinik Araştırmalar Etik Kurulu'dan 11/05/2020 tarih ve HRU/20.09.08 sayılı kararı ile alınmıştır. Hasta görüntüleri aksiyel, koronal ve saggital planlarda incelendi. Hasta görüntülerinin dahil edilme kriterleri şöyledir; 1) Her iki maksiller sinüste net bir şekilde incelenebiliyor olmalı. 2) Herhangi bir patoloji veya herhangi bir yabancı cisim olmamalı. 3) Daha önce maksiller sinüse yönelik herhangi bir cerrahi uygulanmamış olmalı.

Görüntülemede Ge's Revolution ct 256-slice scanner cihazı kullanılmıştı. Görüntüler $0.6 \mathrm{~mm}$ aksiyel, koronal ve sagital kesitlerle incelenmiştir.

Çalışma 500 adet baş boyun bölgesi bilgisayarlı tomografi görüntüsü, değerlendirilerek gerçekleştirildi. Görüntüler incelenirken her hasta için, hastanın yaşı, cinsiyeti, hastanın dişli-kısmi dişli-tam dişsiz oluşu parametreleri kaydedildi. Septalar koronal, sagital ve transversal olmak üzere sınıflandırıldı (14)( Şekil 1a, 1b, 1c). Ayrıca septanın bulunduğu konuma göre; anterior (kanin dişin distalinden ikinci premolar dişin distaline), orta üçlü (birinci molar dişin mezyalinden ikinci molar dişin distaline), ve posterior (ikinci molar dişin distalinden maksiler sinüsin distal duvarına) olmak üzere 3 sınıfa ayrıldı.
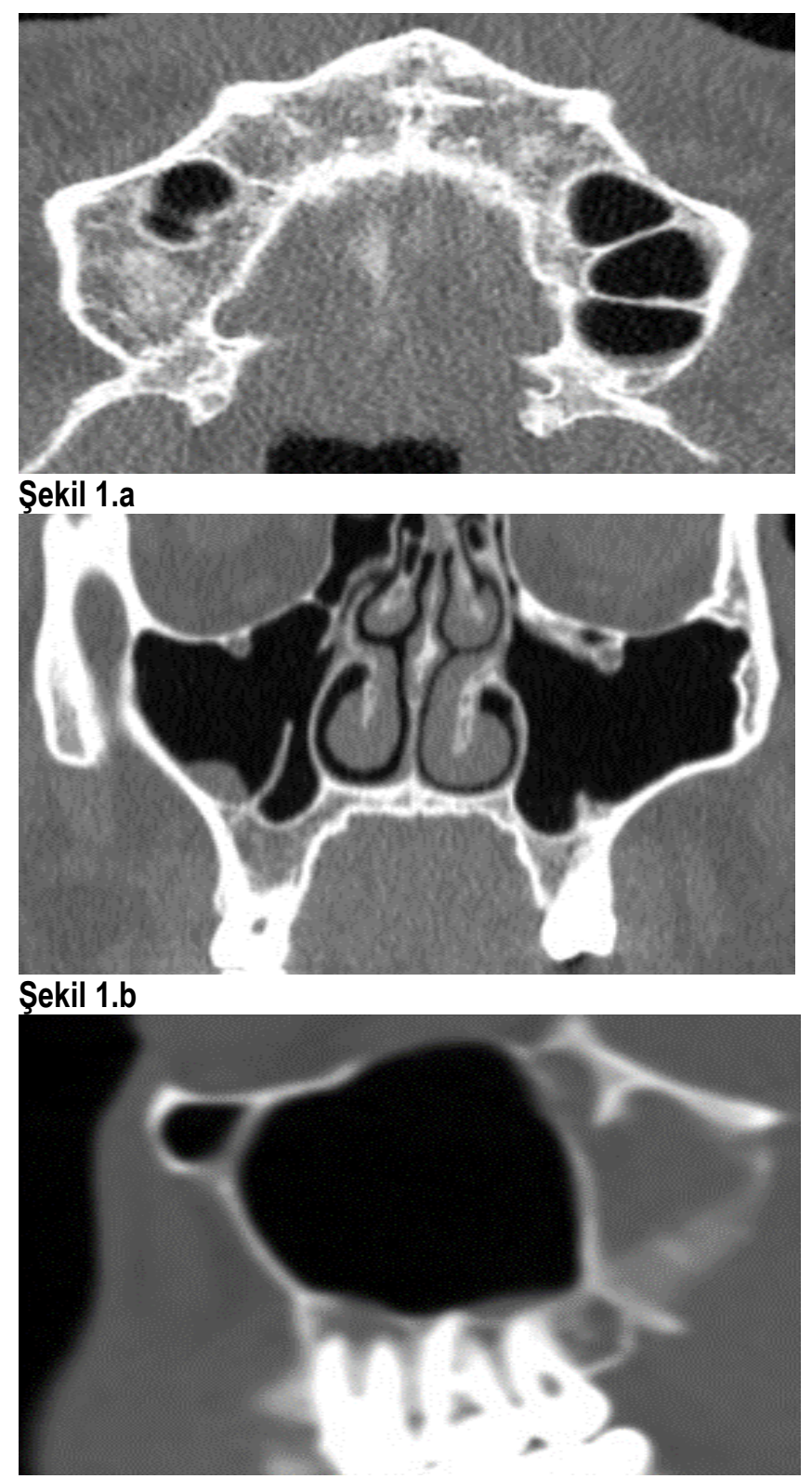

Şekil 1.c

Şekil 1. Hastaların CT görüntüleri

1a) Septanın koronal yönelimi

1b) Septanın sagital yönelimi

1c) Septanın transversal yönelimi 
Toplanan verilerin analizi, SPSS 20.0. istatistiksel yazilım paketi kullanılarak yapıldı. Illk once tanımlayıcı istatistik uygulandı. Maksiller sinus septasının hasta ile ilgili faktörleri olan yaş, cinsiyet ve dentisyon tipi ile ilişkisini değerlendirmek içi Chi-square testi kullanıldı. İstatistiksel anlamlılık sınırı olarak $p<0.05$ kabul edildi.

\section{Bulgular}

Toplam 500 adet bilgisayarlı tomografi görüntüsü incelendi. Bunların 446 tanesi dahil edilme kriterlerine uyduğundan çalışmaya dahil edildi. Böylece toplam 892 tane maksiller sinüs incelendi. İncelenen hastaların 198'i (\%44.4) erkek ve 248'i (\%55.6) kadındı. Hastaların ortalama yaşı 33.4, en küçüğü 18, en büyüğü 79 yaşındaydı. Hastaların 248'i (\%55.6) tam dişli, 168'i(\% 37.7) kısmi dişli ve 30'u (\%6.7) tam dişsizdi.

135 (\% 30.2) hastada en az bir septum tespit edildi. Bu hastaların 126 'sında (\% 93.3) sadece bir septum görülürken, 9'unda (\% 6.7) birden fazla septum görüldü. Hastalarda toplam 146 adet septum tespit edildi. Maksiller sinüs septa prevelansı ile hastaların yaşı $(p=0.415)$, cinsiyeti ( $p$ $=0.443)$ ve dentisyon tipi $(p=0.078)$ arasında anlamlı bir ilişki bulunamamıştır.

146 septanın 83 tanesi (\% 56.8) koronal, 35 tanesi (\% 24) sagittal ve 28 tanesi (\% 19.2) transversal yöndeydi. Koronal olarak yönelenlerin 14 tanesi (\%16.9) anteriorda, 45 tanesi (\%54.2) orta üçlüde ve 24 tanesi (\%28.9) posteriordaydı. Sagittal olarak yönelenlerin 16 tanesi (\%45.7) anteriorda, 10 tanesi (\%28.6) orta üçlüde ve 9 tanesi (\%25.7) posteriordaydı. Transversal olarak yönelenlerin 9 tanesi (\%32.1) anteriorda, 11 tanesi (\%39.3) orta üçlüde ve 8 tanesi (\%28.6) posteriordaydı. Septanın yönelim ve konumları arasında anlamlı bir ilişki bulunmuştur $(p=0.017)$. Septanın yönelim ve konumları Tablo 1'de gösterilmiştir.

\section{Tartışma}

Maksiller sinus bölgesine planlanan cerrahilerde, maksiller sinüsin anatomik oluşumlarının ve varyasyonlarının preoperative belirlenmesi oldukça önemlidir. Özellikle tomografilerle bu oluşumların üç boyutlu olarak değerlendirilmesi cerrahinin başarısını önemli ölçüde arttırır ve oluşabilecek komplikasyonlar için önlem alınabilir. Çünkü maksiller sinus içerisindeki anatomik veya patolojik oluşumlar deneyimli cerrahları bile oldukça zorlamaktadır (5-7).

Maksiller sinüs septası sinüs içersinde kortikal kemikten oluşmuş, maksiller sinüsü iki veya daha fazla bölüme ayıran ve sinüsün kemik dayanıklıığını artıran ince bir duvardır. Maksiller sinüs septa varlığı, cerrahi sırasında Schneiderian membrane perforasyonuna bağlı akut veya kronik sinüzite veya yerleştirilen greftin rezorpsiyonuna neden olabilir (4).

Farklı toplumlarda yapılan çalışmalarda maksiller sinüs septası insidansı, konumu ve yönelimi değişen oranlarda bildirilmiştir. Bu çalışmada 446 hastanın $135^{\circ}$ inde (\% 30.2) toplam 146 adet septum varlığı saptanmıştır. Bu oran, maksiller sinüs septum varlığına göre, Underwood' un \% 33,11 Neugebauer ve ark' in \% 33.2,12 Rosano ve ark' in \% 33.3,13 Köymen ve ark' in \% 35.4,14 Qianveark'ın \% 36.2,15 Naitohve ark' In \% 37,16 olarak bildirdiği sonuçlarıyla uyumlu bulunmuştur (15-20).

Tablo 1. Maksiller sinüs septasının yönelim ve konumları

\begin{tabular}{|c|c|c|c|c|c|c|c|}
\hline & & \multicolumn{6}{|c|}{ KONUM } \\
\hline & & \multicolumn{2}{|c|}{ ANTERIOR } & \multicolumn{2}{|c|}{ ORTA ÜÇLÜ } & \multicolumn{2}{|c|}{ POSTERIOR } \\
\hline & & Adet & Yüzdelik & Adet & Yüzdelik & Adet & Yüzdelik \\
\hline \multirow{3}{*}{ 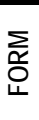 } & KORONAL & 14 & $16,9 \%$ & 45 & $54,2 \%$ & 24 & $28,9 \%$ \\
\hline & SAGGITAL & 16 & $45,7 \%$ & 10 & $28,6 \%$ & 9 & $25,7 \%$ \\
\hline & TRANSVERSAL & 9 & $32,1 \%$ & 11 & $39,3 \%$ & 8 & $28,6 \%$ \\
\hline
\end{tabular}

Yapılan farklı çalışmalarda maksiller sinüs insidansı ile hastaların yaşı ve cinsiyeti arasında anlamlı ilişki bulunamamıştır. Ancak maksiller sinüs insidansı ile dentisyon tipi arasındaki ilişki açısından birbirinden farklı sonuçlar bulunmuştur. Yapılan bazı çalışmalarda maksiller sinüs septa insidansı, dişsiz hastalarda daha yüksek bulunmuşken, bazılarında kısmi dişli hastalarda daha yüksek bulunmuştur (21-24). Bazı çalışmalarda da maksiller sinüs septa insidansı ile dentisyon tipi arasında anlamlı bir ilişki bulunamamıştır (25).Çalışmamızda maksiller sinüs insidansı ile hastaların yaşı $(p=0.415)$, cinsiyeti $(p=0.443)$ ve dentisyon tipi $(p=0.078)$ arasında anlamlı bir ilişki bulunamamıştır. Maksiller sinüs septasının en yaygın bulunduğu konumu Underwood posterior olarak bildirmesine ragmen, Krennmair anterior olarak bildirmiştir $(8,21)$. Pommer ve arkadaşları sinüs septasının en yaygın bulunduğu konumu \% 54.6'lık oranla molar dişler bölgesi olan orta bölgede bulmuşlardır (26). Buna benzer olarak yaptığımız çalışmada, \% 45.2 ile maksiller sinüsün en yaygın septum lokalizasyonu orta bölgede bulunmuştur ve bunu sırasıyla \% 28.1 ile arka ve \% 26.7 ile ön bölge izlemiş̧ir. Maksiller sinüs septasının yöneliminde farklı araştırmacılar farklı sınıflandırmalar kullanmışlardır. Çalışmamızda maksiller sünüsin yöneliminde A. Hungerbu"hler ve arkadaşlarının sınıflamasına benzer bir sınıflama kullandık (14). Septa yönelimi en çok \% 56.8 koronal ve sırasıyla \% 24 sagittal ve \% 19.2 transversal yönde izlemiştir. Septanın yönelim ve konumları arasında anlamlı bir ilişki bulunmuştur ( $p=0.017)$.

Çalışmamızda; çalışmanın retrospektif olması, tek gözlemci tarafından taranması gibi bazı kısıtlayıcı faktörler bulunmaktaydı.Bu kısıtlamalara ragmen şu sonuçlar çıkarılabilir: 1) Maksiller sinüs septa prevelansı ile hastaların yaşı $(p=0.415)$, cinsiyeti $(p=0.443)$ ve dentisyon tipi $(p=$ 0.078 ) arasında anlamlı bir ilişki bulunamamışıtır. 2) Maksiller sinüse yönelik herhangi bir cerrahi planlamasında, maksiller sinüs septa sayısı, morfolojisi, lokalizasyonu ve yönelimi komplikasyonları önlemede etkili olacaktır.

$\mathrm{Bu}$ çalışmanın sonucunda maksiller sinüs septasının po- 
pülasyonun yaklaşık üçte birinde olduğu görüldü. Yapılacak implant cerrahilerinin üç boyutlu görüntülerle planlanması yaşanabilecek komplikasyonların azalmasını, hatta implant planlamasını iki boyutlu görüntülere göre değiştirebileceği görüldü. Ancak bu çalışma sonuçlarının yapılacak daha kapsamlı çalışmalarla doğrulanmasına intiyaç olduğu değerlendirildi.

\section{Açıklamalar}

Etik Onam: Harran Üniversitesi Klinik Araştırmalar Etik Kurulu'dan 11/05/2020 tarih ve HRU/20.09.08 sayılı kararı ile alınmıştır

\section{Kaynaklar}

1. Tadinada A, Fung K, Thacker S, Mahdian M, Jadhav A, Schincaglia GP. Radiographic evaluation of the maxillary sinus prior to dental implant therapy: a comparison between two-dimensional and three-dimensional radiographic imaging. Imaging SciDent 2015; 45: 169-74.

2. Balshi TJ, Wolfinger GJ. Management of the posterior maxilla in the compromised patient: historical, current, and future perspectives. Periodontol 2000 2003; 33: 67-81.

3. Al-Dajani M. Recent trends in sinus lift surgery and their clinical implications. Clin Implant Dent Relat Res 2016;18:204-12.

4. Schwarz L, Schiebel V, Hof M, Ulm C, Watzek G, Pommer B. Risk factors of membrane perforation and postoperative complications in sinus floor elevation surgery: review of 407 augmentation procedures. J Oral Maxillofac Surg 2015;73:1275-82.

5. Al-Dajani M. Incidence, risk factors, and complications of Schneiderian membrane perforation in sinus lift surgery: a meta analysis. Implant Dent 2016;25:409-15.

6. Toscano NJ, Holtzclaw D, Rosen PS. The effect of piezoelectric use on open sinus lift perforation: a retrospective evaluation of 56 consecutively treated cases from private practices. J Periodontol2010;81:16771.

7. Becker ST, Terheyden H, Steinriede A, Beh- rens E, Springer I, Wiltfang J. Prospective observation of 41 perforations of the Schneiderian membrane during sinus flor elevation. Clin Oral Implants Res 2008;19:1285-9.

8. Underwood AS (1910) An inquiry into the anatomy and pathology of the maxillary sinus. J Anat Physio 144:354-369

9. Rancitelli D, Borgonovo AE, Cicciu M, ReD, Rizza F, Frigo AC, Maiorana C. Maxillaryinus septa and anatomic correlation with the Schneiderian membrane. J Craniofac Surg 2015;26:1394-8.

10. Maestre-Ferrin L, Galan-Gil S, Rubio-Serrano M, Penarrocha-Diago M, Penarrocha-Oltra D. Maxillary sinus septa: a systematic review. Med Oral Patol Oral Cir Bucal 2010;15:e3836.

11. Velasco-Torres M, Padial-Molina M, Avila-Ortiz G, Garcia-Delgado R, O'Valle F,CatenaA, Galindo-Moreno P. Maxillary sinus dimensions decrease as age and tooth loss increase. Implant Dent 2017;26:28895.

12. Vinter I, Krmpotic-Nemanic J, Hat J, Jalsovec D. Does the alveolar process of the maxilla always disappear after tooth loss? Laryngo rhino otologie 1993;72:605-7.

13. Greenberg AM. Digital Technologies for dental implant treatment planning and guided surgery. Oral Maxillofac Surg Clin North Am 2015;27:319-40.

14. A. Hungerbu"hler, C. Rostetter, H.-T. Lu"bbers, M. Rücker, B. Stadlinger: Anatomical characteristics of maxillary sinus septa visualized by cone beam computed tomography. Int. J. Oral Maxillofac. Surg. 2019; 48: 382-387.

15. Underwood AS, Surgical considerations connect with the anatomy of the maxillary sinus. Br Med J 1909;15: 1.
16. Neugebauer J, Ritter L, Mischkowski RA, Dreiseidler T, Scheer $P$, Ketterle M, Rothamel D, Zöller JE. Evaluation of maxillary sinus anatomy by cone-beam CT prior to sinus flor elevation. Int J Oral Maxillofac Implants 2010;25: 258-65.

17. Rosano G, Taschieri S, Gaudy JF, Lesmes D, Del Fabbor M Maxillary sinus septa: a cadaveric study. J Oral Maxillofac Surg 2010;68:1360-64.

18. Koymen R, Gocmen Mas N, Karacayli U, Ortakoglu K, Ozen T, Yazici AC. Anatomic evaluation of maxillary sinussepta: surgery and radiology. Clin Anat 2009;22:563-70.

19. Qian L, Tian XM, Zeng L, Gong Y, Wei B. Analysis of the Morphology of Maxillary Sinus Septa on Reconstructed Cone-Beam Computed Tomography Images. J Oral Maxillofac Surg 2016;74:729-37.

20. Naitoh M, Suenega $Y$, Kondo S, Gotoh K, Arijı E. Assessment of maxillary sinus septa using cone-beam computed tomography: etiological consideration. Clin Implant Dent Releated Res 2009;11: 52-8.

21. Krennmair G, Ulm CW, Lugmayr H, Solar P. The incidence, location, and height of maxillary sinus septa in the edentulous and dentate maxilla. J Oral Maxillofac Surg. 1999;57:667-671. discussion 671-662.

22. Schriber $M$, von Arx $T$, Sendi $P$, et al. Evaluating maxillary sinus septa using cone beam computed tomography: is there a difference in frequency and type between the dentate and edentulous posterior maxilla? Int J Oral Maxillofac Implants. 2017; 32(6): 1324-1332, doi: 10.11607/jomi.5854, indexed in Pubmed: 29140376.

23. Orhan K, Kusakci Seker B, Aksoy S, Bayindir H, Berberoğlu A, Seker E. Cone beam CT evaluation of maxillary sinus septa prevalence, height, location and morphology in children and an adult population. Med Princ Pract 2013;22(1):47-53

24. Ozeç I, Kılıç E, Müderris S. Maxillary sinus septa: Evaluation with computed tomography and Panoramic radiography. Cumhuriyet Dental Journal 2008;11:82-86

25. N. Dedeoğlu, O. Altun. Evaluation of maxillary sinus anatomical variations and pathologies in elderly, young, posterior dentate and edentulous patient groups with cone-beam computed tomography Folia Morphol.Vol. 78, No. 3, pp. 595-599 DOI: 10.5603/FM.a2019.0013

26. Pommer B, Ulm C, Lorenzoni M, Palmer R, Watzek G, Zechner W.Prevalence, location and morphology of maxillary sinus septa: systematic review and meta-analysis. J Clin Periodontol. 2012;39:769-773. 